\title{
Atomic emission detector with gas chromatographic separation and cryogenic pre-concentration (CryoTrap-GC-AED) for atmospheric trace gas measurements
}

Einar Karu ${ }^{1}$, Mengze Li $^{1}$, Lisa Ernle ${ }^{1}$, Carl A.M. Brenninkmeijer ${ }^{1}$, Jos Lelieveld ${ }^{1}$, Jonathan Williams ${ }^{1}$

$5 \quad{ }^{1}$ Atmospheric Chemistry Department, Max Planck Institute for Chemistry, 55128 Mainz, Germany

Correspondence to: Jonathan Williams (jonathan.williams@mpic.de)

\section{Supplementary}

Apel-Riemer-2015, 84 multi-component gas calibration standard

Table S1: Retention times of Apel-Riemer-2015, Apel-Riemer Environmental, Inc. 84 multi-component gas phase calibration mix in

10 UHP nitrogen balance. Stated uncertainty better than $\pm 5 \%$ for all components. MR certificate analysis date: June 9, 2015 .

\begin{tabular}{llll}
\hline IAGOS- & & & \\
CARIBIC-2018 & & CAS \# & $\begin{array}{l}\text { Concentration } \\
\text { (ppb) }\end{array}$ \\
\hline RT 30 ${ }^{\circ} \mathbf{C}(\mathbf{m i n})$ & Compound & $115-07-1$ & 51.8 \\
\hline 3.46 & Propene & $463-58-1$ & 51.2 \\
3.50 & Carbonyl Sulfide (OCS) & $75-71-8$ & 49.6 \\
3.55 & Dichlorodifluoromethane (R-12) & $75-45-6$ & 50.8 \\
3.61 & Chlorodifluoromethane (HCFC-22) & $76-14-2$ & 55.5 \\
3.91 & 1,2-Dichlorotetrafluoroethane (R-114) & $74-87-3$ & 52.5 \\
4.06 & Chloromethane & $115-11-7$ & 51.3 \\
4.31 & Isobutene & $75-01-4$ & 52.3 \\
4.39 & Vinyl Chloride & $106-99-0$ & 51.7 \\
4.50 & $1,3-B u t a d i e n e$ & $75-07-0$ & 58.2 \\
4.77 & Acetaldehyde & $67-56-1$ & 51.7 \\
5.20 & Methanol & $74-83-9$ & 55.0 \\
5.38 & Bromomethane & $75-00-3$ & 54.8 \\
5.72 & Chloroethane & $75-69-4$ & 51.8 \\
6.50 & Trichlorofluoromethane (F-11) & $109-66-0$ & 61.8 \\
6.77 & Pentane & $64-17-5$ & 45.3 \\
& Ethanol & &
\end{tabular}


1,1,2-Trichloro-1,2,2-Trifluoroethane (CFC-113)

76-13-1 $\quad 70.6$

Methyl Iodide

74-88-4 $\quad 69.4$

Carbon Disulfide $\left(\mathrm{CS}_{2}\right)$

$75-15-0 \quad 47.0$

9.44

2-Propanol

67-63-0 $\quad 50.7$

Acetonitrile

75-05-8 50.0

Dichloromethane

75-09-2 52.2

Cyclopentane

287-92-3 $\quad 52.8$

11.14

Acrylonitrile

trans-1,2-Dichloroethene

$107-13-1 \quad 64.3$

Methyl Tertiary Butyl Ether (MTBE)

11.82

Hexane

$156-60-5 \quad 62.3$

12.25

Methacrolein

12.36

12.58

12.93

13.57

1,1-Dichloroethane

1634-04-4 61.0

110-54-3 52.1

13.66

Vinyl Acetate

1-Propanol

78-85-3

48.9

14.05

Butanal

75-34-3

57.8

108-05-4 56.4

71-23-8 $\quad 51.4$

123-72-8 59.6

Methyl Vinyl Ketone

78-94-4 74.4

cis-1,2-Dichloroethene

156-59-2 52.3

14.10

Methyl Ethyl Ketone

78-93-3

51.1

14.90

15.32

Chloroform

67-66-3 51.9

1,1,1-Trichloroethane

Cyclohexane

71-55-6

Tetrachloromethane

110-82-7 53.4

16.18

Benzene

56-23-5 51.4

16.36

1,2-Dichloroethane

71-43-2

48.1

107-06-2 58.5

Trichloroethylene

79-01-6 $\quad 54.8$

17.83

1-Butanol

71-36-3

40.5

18.04

Isopropyl nitrate 
18.31

18.40

18.56

18.64

18.69

19.07

19.81

20.14

20.47

20.85

21.33

21.49

21.90

22.14

22.44

22.68

23.06

24.21

24.42

24.71

25.66

25.70

26.16

27.34

27.96

28.88

29.61

29.84

29.91

30.15
Hydroxyacetone

2-Pentanone

1,2-Dichloropropane

Pentanal

3-Pentanone

1,4-Dioxane

Bromodichloromethane

Propyl nitrate

cis-1,3-Dichloropropene

4-Methyl-2-Pentanone

Toluene

trans-1,3-Dichloropropene

1,1,2-Trichloroethane

Isobutyl nitrate

3-Hexanone

Tetrachloroethylene

2-Hexanone

Hexanal

1,2-Dibromoethane

Chlorobenzene

Ethyl Benzene

m-Xylene

p-Xylene

o-Xylene

Styrene

Bromoform

1,1,2,2-Tetrachloroethane

1,3,5-Trimethylbenzene

1,2,4-Trimethylbenzene

(m-)1,3-Dichlorobenzene

(p-)1,4-Dichlorobenzene

1,2,3-Trimethylbenzene

Benzyl Chloride

$\begin{array}{ll}116-09-6 & 53.1 \\ 107-87-9 & 50.2 \\ 78-87-5 & 50.5 \\ 110-62-3 & 59.9 \\ 96-22-0 & 52.5 \\ 123-91-1 & 50.7 \\ 75-27-4 & 49.2 \\ 627-13-4 & 47.4 \\ 10061-01-5 & 49.6 \\ 108-10-1 & 51.7 \\ 108-88-3 & 51.6 \\ 10061-02-6 & 51.3 \\ 79-00-5 & 52.9 \\ 543-29-3 & 45.0 \\ 598-38-8 & 50.9 \\ 127-18-4 & 55.5 \\ 591-78-6 & 52.0 \\ 66-25-1 & 55.8 \\ 106-93-4 & 50.2 \\ 108-90-7 & 53.3 \\ 100-41-4 & 50.8 \\ 108-38-3 & 50.8 \\ 106-42-3 & 51.5 \\ 95-47-6 & 50.6 \\ 100-42-5 & 52.5 \\ 75-25-2 & 52.4 \\ 79-34-5 & 50.7 \\ 108-67-8 & 51.2 \\ 95-63-6 & 50.7 \\ 541-73-1 & 52.9 \\ 106-46-7 & 55.6 \\ 526-73-8 & 46.2 \\ 100-44-7 & 61.7 \\ & \\ 10\end{array}$


NPL-2017, National Physical Laboratory (NPL) 30 component NMHC primary calibration standard

Table S2: Retention times of NPL-2017 30 ozone precursor NMHC gas phase primary calibration reference material in UHP nitrogen balance. Stated uncertainties are based on $2 \sigma$, providing a coverage probability of $\sim 95 \%$. Calibration date 7 May -21 June

\begin{tabular}{|c|c|c|c|}
\hline \multicolumn{2}{|l|}{$\begin{array}{l}\text { IAGOS- } \\
\text { CARIBIC-2018 }\end{array}$} & \multicolumn{2}{|c|}{ NPL-2017 (Bottle: D51 7546) } \\
\hline RT $30^{\circ} \mathrm{C}(\min )$ & Compound & $\operatorname{MR}\left(\mathrm{pmol} \mathrm{mol}^{-1}\right)$ & $\begin{array}{l}\text { Uncertainty } \\
\left(\text { pmol mol }^{-1}\right)\end{array}$ \\
\hline & Ethene & 3930 & 80 \\
\hline & Ethyne & 4140 & 210 \\
\hline 3.15 & Ethane & 4010 & 80 \\
\hline \multirow{2}{*}{3.46} & Propene & 3930 & 80 \\
\hline & Propane & 3950 & 80 \\
\hline 3.93 & Isobutane & 4030 & 110 \\
\hline 4.31 & 1-Butene & 3980 & 80 \\
\hline 4.36 & n-Butane & 3990 & 80 \\
\hline 4.50 & 1,3-Butadiene & 4040 & 80 \\
\hline 4.60 & trans-2-Butene & 4000 & 80 \\
\hline 4.88 & cis-2-Butene & 3990 & 80 \\
\hline 5.90 & Isopentane & 3940 & 80 \\
\hline 6.58 & 1-Pentene & 4040 & 80 \\
\hline 6.76 & n-Pentane & 3960 & 80 \\
\hline 7.28 & trans-2-Pentene & 3980 & 80 \\
\hline 7.61 & Isoprene & 4140 & 90 \\
\hline 10.11 & 2-Methylpentane & 4150 & 80 \\
\hline 11.81 & n-Hexane & 4150 & 80 \\
\hline 16.17 & Benzene & 4140 & 80 \\
\hline 16.33 & 2,2,4-Trimethylpentane & 3900 & 80 \\
\hline 16.84 & n-Heptane & 4160 & 80 \\
\hline 20.84 & Toluene & 4020 & 110 \\
\hline 21.08 & n-Octane & 3910 & 80 \\
\hline
\end{tabular}


p-Xylene

8460

220

27.95

o-Xylene

4160

110

28.86

1,3,5-Trimethylbenzene

3940

100

29.90

1,4,4-Trimethylbenzene

4060

110

1,2,3-Trimethylbenzene

3890

100 